\title{
Distribución heterogénea de la prevalencia de anticuerpos contra Trypanosoma cruzi en donadores de sangre en Puebla, México
}

Víctor M Monteón, Dr en C, ${ }^{(1)}$ Pedro A Reyes-López, MC, ${ }^{(2)}$ Adalid Sosa-Palacio, Q FB, ${ }^{(2)}$ Gloria León-Tello, M en $C_{,}{ }^{(2)}$ Jaime Martínez-Murguía, MC, ${ }^{(1)}$ Francisca Sosa-Jurado, MSP.(3)

\begin{abstract}
Monteón VM, Reyes-López PA, Sosa-Palacio A, León-Tello G, Martínez-Murguía J, Sosa-Jurado F. Distribución heterogénea de la prevalencia de anticuerpos contra Trypanosoma cruzi en donadores de sangre en Puebla, México.
\end{abstract} Salud Publica Mex 2005;47:116-125.

\section{Resumen}

Objetivo. Identificar la seroprevalencia de anticuerpos anti Trypanosoma cruzi (Ac anti-T. cruzi) en donadores de sangre que habitan en ámbito rural y suburbano, así como las regiones del estado de mayor riesgo y factores asociados. Material y métodos. Estudio transversal realizado de enero a diciembre de 2003. Se analizaron 2489 donadores de sangre reclutados en 10 puestos de sangrado del Instituto Mexicano del Seguro Social (IMSS) distribuidos en las siete regiones económicas del estado de Puebla, México. Se determinó la seroprevalencia mediante las pruebas serológicas obligatorias del panel viral y, además, para T. cruzi, región de reclutamiento y de origen de los donadores. $\mathbf{R e}$ sultados La seroprevalencia de Ac anti-T. cruzi fue de 1.24\% (31/2 489) comparable con la obtenida para el virus de la hepatitis C (1.5\%) y por arriba de la del virus de la inmunodeficiencia humana $(0.4 \%)$ y del antígeno de superficie del virus de la hepatitis B (0.3\%). Las regiones de TehuacánSierra N egra y Mixteca fueron las de mayor riesgo con seroprevalencias, por el origen del donador, de $2.6 \%$ para T. cruzi, mientras que en los originarios de las regiones Sierra nororiental y Angelópolis no se detectaron casos positivos. Se observó asociación entre ser seropositivo y mayor de 40 años y ser originario de las regiones de Tehuacán-Sierra N egra y Mixteca. Conclusiones La distribución de seroprevalencia a T. cruzi es heterogénea, oscila desde $0 \%$ hasta $2.6 \%$, y se reconoce a Tehuacán-Sierra N egra y Mixteca como las regiones de mayor riesgo.

Palabras clave: enfermedad de Chagas; seroprevalencia de T.cruzi; donado res de sangre; México

\author{
Monteón VM, Reyes-López PA, Sosa-Palacio A, \\ León-Tello G, Martínez-Murguía J, Sosa-Jurado F. \\ Heterogeneous distribution of the prevalence \\ of anti-Trypanosoma cruzi antibodies \\ among blood donors in the State of Puebla, Mexico.
} Salud Publica Mex 2005;47:116-125.

\begin{abstract}
A bstract
Objective. To determine the seroprevalence and associated factors, of antibodies against Trypanosoma cruzi (T. cruzi $\mathrm{Ab}$ ) among blood donors living in rural and suburban areas and risk regions. Material and Methods A cross-sectional study was conducted from January to D ecember 2003, in 2489 blood donors of seven regions of Puebla, who were evaluated for mandatory viral and T. cruzi serological tests using validated procedures Results The seroprevalence for T. cruziA b was $1.24 \%$ (31/2489), similar to hepatitis C (HVC) $(1.5 \%)$ and higher than human immunodeficiency virus (HIV) $(0.4 \%)$ and hepatitis B (HVB) $(0.3 \%)$. The highest seroprevalences were observed in the regions of Tehuacan-Sierra $\mathrm{N}$ egra and Mixteca, up to $2.6 \%$, while in Sierra nororiental and Angelopolis no positive blood donors were identified. A positive association was observed between seropositivity and being older than forty years and being born and raised in Tehuacan-Sierra N egra and Mixteca. Conclusions T.cruzi seroprevalence distribution is heterogeneous, from $0 \%$ to $2.6 \%$, with higher seroprevalences in the regions of Tehuacan-Sierra N egra and Mixteca.
\end{abstract}

Key words: Chagas disease; T. cruzi, seroprevalence; blood donors; Mexico

(1) Laboratorio de Inmunoparasitología, Instituto N acional de Cardiología D r. Ignacio Chávez. México, D F, México.

(2) Departamento de Microbiología, Facultad de Ciencias Q uímicas, Benemérita Universidad Autónoma de Puebla, México.

(3) Hospital de Especialidades del Centro Médico N acional Manuel Avila Camacho, Instituto Mexicano del Seguro Social, Puebla, México.

Fecha de recibido: 2 de agosto de 2004 • Fecha de aceptado: 10 de febrero de 2005

Solicitud de sobretiros: Francisca Sosa Jurado. Hospital de Especialidades, Centro Médico Nacional Manuel Avila Camacho, Instituto Mexicano del Seguro Social. 2 N orte N 0.2004, colonia Centro, 72000 Puebla, Puebla, México.

Correo electrónico: sosajurado@ hotmail.com 
a tripanosomosis americana (TA) es causada por el protozoario Trypanosoma cruzi, transmitido entre animales y humanos por triatominos que, al momento de alimentarse de sangre, expulsan tripanosomas en sus deyecciones, que penetran a través de la herida causada por la picadura, por el rascado, otras abrasiones de la piel o de las mucosas y que afectan diversos órganos, principalmente corazón, esófago y colon. ${ }^{1}$

La infestación de las viviendas por triatominos pone en riesgo de adquirir la infección a un mínimo de 90 millones de personas en América Latina y se estima que 16 a 18 millones de personas están infectadas con T. cruzi. La TA ha sido considerada como una enfermedad del medio rural, pero los cambios socioeconómicos han hecho que se incremente la migración de las personas de las zonas rurales endémicas a las zonas urbanas, en donde no existen los vectores, y de esta forma urbanizar la transmisión de la infección a través de la donación de sangre de sujetos infectados pero asintomáticos. Así, se considera a la transfusión sanguínea como el segundo mecanismo más importante para la transmisión de T. cruzi, después del vectorial. ${ }^{2}$

El primer estudio realizado en México para determinar anticuerpos contra T. cruzi (Ac anti-T. cruzi) en donadores de sangre se realizó en el estado de Oaxaca, en 1978, y se obtuvo una seroprevalencia de $4.4 \%{ }^{3} \mathrm{El}$ primer caso de transmisión por transfusión sanguínea se informó en 1989. ${ }^{4}$

En México la Norma Oficial Mexicana 003-SSA21993 establece la realización de pruebas serológicas en donadores de banco de sangre en las zonas endémicas, pero no se precisa cuáles son éstas. ${ }^{5}$ De forma independiente se han realizado diversos estudios seroepidemiológicos en distintas regiones del país, entre ellos el trabajo llevado a cabo en 1991 con una muestra de 3419 donadores de sangre, procedentes de 12 municipios rurales y de ocho hospitales urbanos en el estado de Jalisco, que reveló una seroprevalencia de 1.28\%. ${ }^{6}$ En el banco de sangre del Instituto Nacional de Cardiología, localizado en el Distrito Federal, se informó en 1992 y 1999 una seroprevalencia de $0.28 \%$ y 0.3\% respectivamente. ${ }^{7,8}$ En el banco de sangre de Cuernavaca, Morelos, en 1998, se documentó el más alto porcentaje informado para un área urbana hasta la fecha con una seroprevalencia de $17 \%,{ }^{9}$ y un estudio en donadores de sangre de 18 estados del país reveló una seroprevalencia general de $1.5 \%{ }^{10}$

Debe considerarse que todos los productos sanguíneos de un donador seropositivo a T. cruzi, pueden ser infectantes. En 1972, Cerisola y colaboradores ${ }^{11}$ demostraron que T. cruzi se mantiene vivo hasta por tres semanas a bajas temperaturas $\left(4^{\circ} \mathrm{C}\right)$. En un trabajo reciente, Monteón y colaboradores, ${ }^{8}$ en 1999, de- tectaron el ADN del parásito en las fracciones de células rojas y plaquetas de las unidades de sangre de donadores seropositivos a T. cruzi.

El estado de Puebla es considerado como no endémico de la TA ya que no existen informes oficiales de casos crónicos, aunque en los últimos seis años se ha detectado la presencia de casos indeterminados en población abierta con seroprevalencias de entre $10 \mathrm{y}$ $4 \%{ }^{12,13}$ Asimismo, se publicó un trabajo donde se informa una seroprevalencia de $7.7 \%$ en donadores de sangre. ${ }^{14}$ En 2003 se reclutaron 53611 donadores de sangre, de los cuales 25785 provinieron del Instituto Mexicano del Seguro Social (IMSS) el cual cuenta con dos bancos de sangre y 11 puestos de sangrado. La Secretaría de Salud cuenta con el Centro Estatal de la Transfusión Sanguínea (CETS), un banco de sangre y 10 puestos de sangrado (figura 1) distribuidos en las siete regiones económicas del estado. ${ }^{15}$

El propósito de este trabajo fue detectar la seroprevalencia de Ac anti-T. cruzi, identificar cuáles regiones presentan mayor riesgo de donadores seropositivos a dicho protozoario, así como los factores de riesgo asociados.

\section{Material y métodos}

\section{Población estudiada}

Se estudió a 2489 donadores de sangre que habitaban en el medio rural y suburbano del estado de Puebla, México, de enero a diciembre de 2003, éstos fueron diagnosticados como aptos para la flebotomía después de cumplir con los requisitos determinados por la Secretaría de Salud (SSA) de México. ${ }^{5}$ Además, deberían de contar con más de 10 años de radicar en el estado de Puebla, para considerarlos como donadores originarios del estado.

Los donadores se reclutaron en nueve puestos de sangrado y en un banco de sangre del Instituto Mexicano del Seguro Social (IMSS) distribuidos en las siete regiones económicas del estado de Puebla, México. ${ }^{15}$ La Región 1 Sierra Norte colinda con los estados de Hidalgo y norte de Veracruz, la conforman 35 municipios, la Región 2 Sierra Nororiental colinda con el norte del estado de Veracruz y tiene 28 municipios, Región 3 Valle de Serdán, con los estados de Veracruz y Tlaxcala conformada por 31 municipios; la Región 4 Angelópolis, con el estado de Tlaxcala y el Estado de México, tiene 32 municipios; la Región 5 Valle de Atlixco e Izúcar de Matamoros colinda con el estado de Morelos y lo conforman 24 municipios; la Región 6 Mixteca colinda con los estados de Oaxaca, Guerrero y Morelos, con 45 municipios, y la Región 7 Tehuacán 


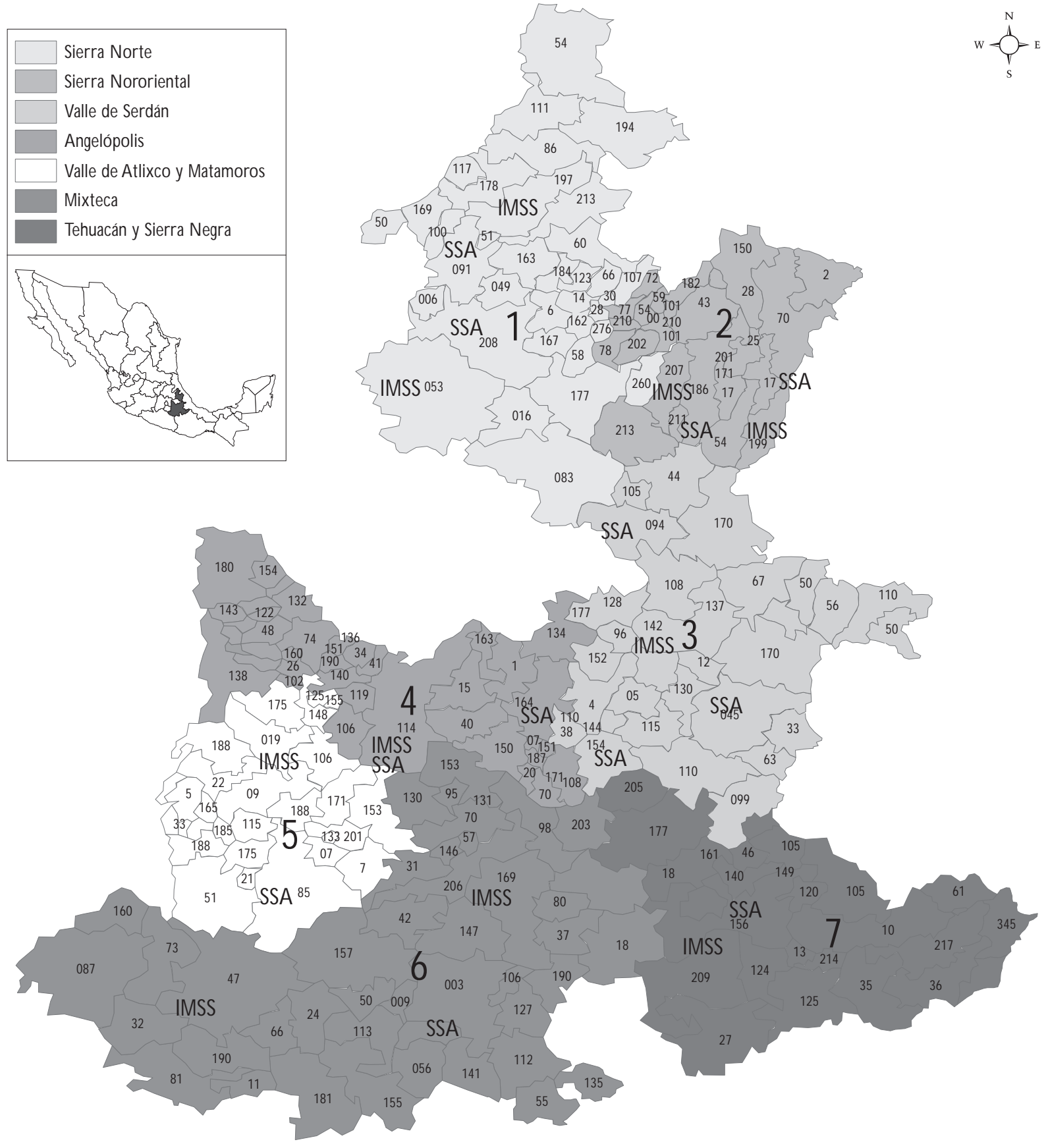

Figura 1. Distribución de Bancos de Sangre (BS) y Puestos de Sangrado (PS) en el Estado de Puebla, México. Del IMSS, R1 Sierra norte: PS Xicotepec de Juárez y Chignahuapan; R2 Sierra Nororiental: PS Zacapoaxtla yTeziutlán; R3Valle de Serdán: PS San Salvador El Seco; R4 Angelópolis: 2 BS, 1PS Puebla; R5Valle de Atlixco e Izúcar de Matamoros: PS Metepec,Atlixco;R6 Mixteca: PS Chiautla deTapia y Tepexi de Rodríguez; R7Tehuacán y Sierra Negra: PS Tehuacán. Por parte de la SSA en la R1 Sierra norte: PS Zacatlán y Huauchinango; R2 Sierra Nororiental: PSTlatlauquitepec yTeziutlán; R3Valle de Serdán: PS Libres y Chalchicomula de Sesna; R4Angelópolis: CeTS Puebla y PSTepeaca; R5Valle de Atlixco e Izúcar de Matamoros: PS Izúcar de Matamoros; R6 Mixteca: PSAcatlán; R7Tehuacán y Sierra Negra: BSTehuacán. 
y Sierra Negra colinda con el estado de Oaxaca y el sur de Veracruz, lo conforman 21 municipios.

\section{Ensayos serológicos}

A cada uno de los 2489 donadores se les tomaron tres muestras sanguíneas con el sistema Vacutainer, una de las cuales fue centrifugada y se separó el suero almacenándolo a $-20^{\circ} \mathrm{C}$, las otras dos se emplearon para determinar hemoglobina, hematocrito, grupo sanguíneo, rastreo de anticuerpos antieritrocitarios y anticuerpos contra treponemas y brucelas. Antes de realizar la flebotomía a los donadores ya seleccionados se les realizó la detección de anticuerpos tipo cardolipina no treponema e inespecíficos (reaginas de la sífilis) con la prueba de floculación VDRL-USR (LICON) y la detección de anticuerpos contra brucelas empleando la prueba de aglutinación cualitativa rosa de Bengala (LICON), ambas pruebas debieron ser negativas para realizar la flebotomía.

Las muestras séricas almacenadas en cada uno de los nueve puestos de sangrado del IMSS fueron enviadas al Banco de sangre del Hospital de Especialidades del Centro Médico Nacional General de División Manuel Avila Camacho, del IMSS, para efectuarles panel viral, que es la determinación de anticuerpos contra virus de la inmunodeficiencia humana (VIH), contra el virus de la hepatitis $\mathrm{C}$ (VHC), anticuerpos contra core del virus de la hepatitis $\mathrm{B}(\mathrm{AcVHBc})$, así como la determinación antígeno de superficie del virus de la hepatitis B (AgVHBs), todos se determinaron con el ensayo enzimoinmunoanálisis de micropartículas (MEIA, por sus siglas en inglés) para detectar anticuerpos o antígenos (Abbot SYSTEM AxSYM). Posteriormente, se enviaron las 2489 muestras séricas al laboratorio de inmunoparasitología del Instituto Nacional de Cardiología (INC) para determinarles anticuerpos contra T. cruzi, con la prueba tamiz ELISA-INC previamente descrita, ${ }^{7}$ la cual consiste en sensibilizar placas de poliestireno con $1 \mu \mathrm{g}$ de antígeno de epimastigotes de T. cruzi cepa INC-1 (cepa mexicana aislada de un caso crónico procedente del estado de Oaxaca), empleando una dilución 1:200 del suero, conjugado anti IgG humano-peroxidasa 1:25 000 y tiempos de incubación de $15 \mathrm{~min}$, a $37^{\circ} \mathrm{C}$. El valor de corte se estableció utilizando 20 sueros de sujetos sanos seronegativos. La densidad óptica (DO) $>0.240$ fue el resultado de la media +5 desviaciones estándar.

La prueba de inmunofluorescencia indirecta (IFIINC), en la cual se empleó como antígeno una suspensión de parásitos de la misma cepa en laminillas preparadas de acuerdo con lo descrito, ${ }^{7}$ consistió en agregar a las laminillas con los parásitos suero diluido 1:32, conjugado anti IgG humano-fluorescencia 1:120, tiempo de incubación $30 \mathrm{~min}$, a temperatura ambiente; se consideraron muestras positivas a diluciones $>1: 32$. En todos los ensayos siempre se incorporaron los controles positivo, negativo y blanco.

\section{Datos generales de los donadores}

Se recabó de las hojas clínicas de cada uno de los donadores de sangre la siguiente información: región, puesto de sangrado de reclutamiento del donador, región de origen del donador, su edad, sexo y ocupación.

Se utilizó el programa epi info versión $6^{\text {a }}$. Se hizo prueba de ji cuadrada corregida de Yates y la exacta de Fisher para determinar la significancia de las asociaciones observadas entre donadores seropositivos a $T$. cruzi y la región de origen del donador, edad, sexo, ocupación, la seropositividad a VIH, VHC, antígeno VHB y la altitud de los municipios. Las variables sometidas a este procedimiento estuvieron dentro de la escala nominal (38 preguntas dicotómicas), el nivel de significancia se situó en $p<0.05$.

\section{Resultados}

\section{Población estudiada}

Se estudiaron 2489 donadores de sangre que habitaban en el ámbito rural y suburbano, de las siete regiones económicas del estado de Puebla, México. ${ }^{15}$ En la Región 1 Sierra Norte se localizan los puestos de sangrado de los Hospitales Rurales Oportunidades (HRO) de Chignahuapan y Xicotepec de Juárez; se reclutaron 406 donadores (15 originarios de otros estados y 24 de otras regiones), 21 de los 35 municipios de esta Región tuvieron algún donador participante en este estudio. En la Región 2 Sierra Nororiental se localizan los puestos de sangrado de HRO-Zacapoaxtla y el Hospital General de Zona (HGZ No. 23) en Teziutlán. Se reclutaron 299 donadores (tres de otros estados, 22 de otra región) y 19 de sus 28 municipios tuvieron algún donador participante. En la Región 3 Valle de Serdán se localiza el puesto de sangrado HRO-San Salvador El Seco donde se reclutaron 92 donadores (16 de otros estados y cinco de otra región) y 22 de sus 31 municipios con donadores. En la Región 4 Angelópolis se localiza el banco de sangre HE CMN MAC, IMSS, en la ciudad de Puebla; ahí se reclutaron 501 donadores (56 de otros estados y 288 de otras regiones) y 24 de sus 32 municipios tuvieron al menos un donador. En la Región 5 Valle de Atlixco e Izúcar de Matamoros se localiza el puesto de sangrado del HGZ No. 5 Metepec, se reclutaron 259 donadores (15 de otros estados y 17 de otras regiones). En la Región 6 Mixteca se localizan los puestos de sangrado de los HRO- Chiautla de Tapia y de Tepexi de 
Rodríguez en donde se reclutaron 245 donadores ( 36 de otros estados y 100 de otras regiones), y en 25 de sus 45 municipios existió algún donador participante. En la Región 7 Tehuacán y Sierra Negra se localiza el puesto de sangrado del HGZ No. 15 de Tehuacán, ahí se reclutaron 687 donadores ( 82 de otros estados y 50 de otras regiones), y 20 de sus 21 municipios tuvieron algún donador. De los 217 municipios que componen el estado de Puebla, 144 de ellos (67\%) tuvieron algún donador de sangre participando en el estudio.

Se reclutaron entre todas las regiones a 223 donadores que fueron originarios de otros estados o bien tenían menos de 10 años de radicar en el estado de Puebla, éstos fueron principalmente de los estados de Veracruz, Morelos, Oaxaca, Tlaxcala, Hidalgo y Chiapas, todos ellos habitaron en ámbito rural; por lo tanto, 2266 donadores fueron originarios del estado de Puebla, México (cuadro I).

\section{Datos generales de los donadores de sangre}

De los 2489 donadores reclutados 264 (10.6\%) fueron mujeres y 2225 (89.4\%) hombres, de lo cual se observó una relación de 1:9.

Su intervalo de edad estuvo entre 18 a 60 años, con una media de 31.4 años, de ellos 1989 (79.9\%) se encontraron entre los 18 a 39 años y los restantes 500 (20.1\%) entre los 40 a 60 años. La mayoría de los donadores reclutados fueron campesinos $1352(54.3 \%)$, trabajadores de maquiladoras, de granjas avícolas o porcícolas y amas de casa; $483(19.4 \%)$, de estas últimas se dedicaron a labores del campo temporalmente, $435(17.5 \%)$, trabajaron en sus pequeñas empresas o en manufacturas que requieren mano de obra calificada, $93(3.7 \%)$ laboraron en el ramo de bienes y servicios, y $125(5.0 \%)$ son profesionales.

\section{Pruebas diagnósticas}

Se obtuvieron 37/2 489 (1.4\%) muestras seropositivas contra T. cruzi por la técnica de ELISA y de éstas, 31 fueron corroboradas como positivas con la técnica de IFI, por lo que se obtuvo una seroprevalencia de casos contra T. cruzi de $1.24 \%$; mientras que $11(0.44 \%)$ fueron seropositivas para $\mathrm{VIH}, 29(1.16 \%)$ para $\mathrm{VHBc}, 38$ $(1.52 \%)$ para VHC y $9(0.36 \%)$ para el AgVHBs.

\section{Distribución de seroprevalencia en las siete regiones del estado}

Al analizar la distribución de la seroprevalencia de acuerdo con la región, obtuvimos para la Región 1
Sierra Norte 5 positivos de los 406 reclutados en ésta $(1.23 \%)$ y, en total, 5 seropositivos de los 377 (1.32\%) que fueron originarios de dicha región y captados ya sea en la misma o en cualquier otra de las regiones. Para la Región 2 Sierra Nororiental hubo 299 donadores y ninguno positivo, en total fueron 383 donadores originarios de ésta, pero la seroprevalencia fue de $0.0 \%$. En la Región 3 Valle de Serdán se detectó a 2 seropositivos de 92 donadores reclutados (2.17\%) y de los 196 donadores originarios de ésta 3 fueron positivos (1.53\%). En la Región 4 de Angelópolis, 4 de 501 donadores reclutados $(0.80 \%)$ fueron seropositivos, y por lugar de origen, ninguno de los 222 originarios de ésta resultaron seropositivos. En la Región 5 Valle de Atlixco e Izúcar de Matamoros, de 259 donadores reclutados $5(1.93 \%)$ fueron seropositivos y de 328 originarios de ésta también hubo 5 seropositivos (1.52\%). En la Región 6 Mixteca se detectó a 5 de 245 donadores reclutados $(2.04 \%)$ y de 115 de los donadores originarios de ésta 3 fueron seropositivos (2.60\%). Por último, para la Región 7 Tehuacán y Sierra Negra, 18 de los 687 donadores reclutados en esta zona fueron seropositivos $(2.62 \%)$ y de 645 donadores originarios de ésta 16 fueron seropositivos (2.48\%). Estas dos últimas regiones fueron las de más alto registro de donadores seropositivos.

De 2489 donadores, 223 (8.9\%) fueron originarios de otros estados y cinco de ellos resultaron seropositivos por ELISA e IFI (2.2\%), dos provinieron del estado de Morelos, dos de Oaxaca y uno de Veracruz. Por lo tanto, hubo 2266 (91.1\%) donadores originarios del estado de Puebla y, de ellos, $26(1.15 \%)$ positivos por dos técnicas serológicas.

Cuando comparamos el nivel de anticuerpos IgG contra T. cruzi de los donadores de sangre seropositivos procedentes del estado de Puebla la DO promedio fue de 0.581 y la de donadores de sangre originarios de otros estados (Morelos, Oaxaca y Veracruz) fue de 0.660, no se encontró diferencia estadísticamente significativa con relación a su procedencia, ni al comparar entre las distintas regiones (cuadro II).

\section{Relación de la seroprevalencia por origen del donador y la altitud de los municipios donde habitaron}

Al comparar las características de altitud de las diferentes regiones y su relación con los sujetos seropositivos encontramos que para la Región 1 Sierra Norte, la altitud de los 21 municipios estudiados se situó entre 190 a $2800 \mathrm{msnm}$, con promedio de 1483 y $5 / 377$ (1.3\%) donadores originarios de esta zona fueron seropositivos (relación 1:66). En la Región 2 Sierra Nororiental, 
la altitud de los 19 municipios fue de entre 700 a 2000 msnm, con promedio de 1267 , y ninguno de los 383 donadores fue seropositivo. En la Región 3 Valle de Serdán, la altitud fue de entre 1950 a $2600 \mathrm{msnm}$, promedio de 2340, y 3 de 196 (1.5\%) fueron seropositivos a T. cruzi (relación 1:65). En la Región 4 Angelópolis la altitud de los 24 municipios estudiados se situó entre los 1950 a $2600 \mathrm{msnm}$, con promedio de 2375 , y ninguno de los 222 donadores originarios de ésta resultó seropositivo. En la Región 5 Valle de Atlixco y Matamoros la altitud de los 22 municipios fue de entre los 1080 a $1850 \mathrm{msnm}$, con promedio de 1075 $\mathrm{msnm}, \mathrm{y}$ 5/328 (1.5\%) donadores originarios de la zona fueron seropositivos a T. cruzi (relación 1:65). En la Región 6 Mixteca, el intervalo de altitud de los 25 municipios fue de entre 800 a $2000 \mathrm{msnm}$, con promedio de $1352 \mathrm{msnm}$, y 3/115 (2.6\%) donadores originarios de la zona fueron seropositivos (relación 1:38). En la Región 7 Tehuacán y Sierra Negra, el intervalo de altitud de los 20 municipios fue de entre 1110 a $2200 \mathrm{msnm}$, con promedio de $1645, \mathrm{y} 16 / 645$ (2.4\%) donadores fueron seropositivos (relación 1:40). Los originarios de estas dos últimas regiones mostraron la mayor seroprevalencia (cuadro III).

\section{Determinación de asociaciones}

La relación de donadores seropositivos y sexo fue de $\chi^{2}=2.77$ con $p=0.088$, por lo que el ser donador seropositivo a T. cruzi es indistinto de ser hombre o mujer.

En cuanto a la relación de donadores seropositivos con el grupo de edad, se observó en el de 40 a 49 años una seroprevalencia de $2.89 \%$, y se obtuvo una $\chi^{2}=12.88$

\section{Cuadro I \\ Ubicación de puestos de sangrado del Instituto Mexicano del Seguro Social y donadores reclutados originarios de las siete regiones económicas del estado de Puebla, México, 2003}

\begin{abstract}
Nombre de la Región (Número de municipios de origen de los donadores / número de municipios que la conforman)
\end{abstract}

1. Sierra N orte (21 de 35)

\author{
Ubicación de puestos \\ de sangrado
}

$\begin{array}{ccc}\text { Donadores } & \text { Donadores } & \text { Donadores originarios } \\ \text { reclutados } & \text { originarios } & \text { de otra región } \\ \text { en la } & \text { de otros } & \text { diferente a la de } \\ \text { región } & \text { estados } & \text { su reclutamiento }\end{array}$

406

HR-O portunidades en los municipios de Xicotepec de Juárez y Chignahuapan

\begin{tabular}{|c|c|c|c|c|c|c|}
\hline 2. Sierra N ororiental (19 de 28) & $\begin{array}{l}\text { HR-O portunidades en los muni- } \\
\text { cipios de Zacapoaxtla y el Hos- } \\
\text { pital General de Zona N } 0.23 \text { en } \\
\text { Teziutlán }\end{array}$ & 299 & 3 & 22 & 109 & 383 \\
\hline 3. Serdán (22 de 31) & $\begin{array}{l}\text { HR-0 portunidades en San Salva- } \\
\text { dor El Seco }\end{array}$ & 92 & 16 & 5 & 125 & 196 \\
\hline 4.Angelópolis* (24 de 32) & $\begin{array}{l}\text { Banco de sangre del HE CMN } \\
\text { MAC en Puebla, Puebla }\end{array}$ & 501 & 56 & 288 & 65 & 222 \\
\hline $\begin{array}{l}\text { 5.Valle de Atlixco e Izúcar de } \\
\text { Matamoros (13 de 24) }\end{array}$ & $\begin{array}{l}\text { Hospital General de Zona N } 0.5 \\
\text { en Metepec, Atlixco }\end{array}$ & 259 & 15 & 17 & 101 & 328 \\
\hline 6. Mixteca (25 de 45) & $\begin{array}{l}\text { HR-0 portunidades en los muni- } \\
\text { cipios de Chiautla de Tapia y Te- } \\
\text { pexi de Rodríguez }\end{array}$ & 245 & 36 & 100 & 6 & 115 \\
\hline $\begin{array}{l}\text { 7. Tehuacán y Sierra N egra ( } 20 \\
\text { de 21) }\end{array}$ & $\begin{array}{l}\text { Hospital General de Zona N } 0.15 \\
\text { en Tehuacán }\end{array}$ & 687 & 82 & 50 & 90 & 645 \\
\hline Total $144(67 \%)$ de 216 (100\%)* & $\begin{array}{l}N \text { ueve puestos de sangrado y un } \\
\text { banco de sangre }\end{array}$ & 2489 & 223 & 506 & 506 & 2266 \\
\hline
\end{tabular}

HE CMN MAC: Hospital de Especialidades del Centro Médico N acional General de División Manuel Avila Camacho 


\section{Cuadro II \\ Seroprevalencia a T. CRuzi de los donadores de SANGRe, por Región de ReClutamiento y por su región de origen. Puebla, México, 2003}

\begin{tabular}{|c|c|c|c|}
\hline Región & $\begin{array}{l}\text { Seroprevalencia por región } \\
\text { de reclutamiento del donador, } \\
\text { empleando la técnica de ELISA }\end{array}$ & $\begin{array}{l}\text { Seroprevalencia por región } \\
\text { de origen del donador, } \\
\text { empleando la técnica de ELISA }\end{array}$ & $\begin{array}{l}\text { Promedio del nivel } \\
\text { de anticuerpos lgG contra } \\
\text { T. cruzi Densidad Optica }\end{array}$ \\
\hline 1. Sierra N orte & $5 / 406(1.2)$ & 5 / 377 (1.3\%) & 0.598 \\
\hline 2. Sierra N ororiental & $0 / 299(0.0)$ & $0 / 383(0.0 \%)$ & \\
\hline 3. Serdán & $2 / 92(2.1)$ & $3 / 196(1.5 \%)$ & 0.582 \\
\hline 4.Angelópolis & $4 / 501(0.8)$ & $0 / 222(0.0 \%)$ & \\
\hline 5.Valle de Atlixco y Matamoros & $5 / 259(1.9)$ & $5 / 328(1.5 \%)$ & 0.542 \\
\hline 6. Mixteca & $5 / 245(2.0)$ & $3 / 115(2.6 \%)$ & 0.588 \\
\hline 7.Tehuacán y Sierra N egra & $16 / 687(2.3)$ & $16 / 645(2.4 \%)$ & 0.536 \\
\hline 8. 0 tros estados & & $5 / 223(2.2 \%)$ & 0.660 \\
\hline & $37 / 2489$ (1.4) & 32 / 2266 (1.41\%) & $\bar{X}=0.581$ \\
\hline
\end{tabular}

ELISA: Enzime Linked Immunosoibant Assay

IFI: inmunofluorescencia indirecta

con $p=0.003$ y para el grupo de 50 a 59 años una seroprevalencia de $8.53 \%$ o una $\chi^{2}=9.33$ con $p=0.021$, encontrándose una asociación con estos grupos de edad.

Al comparar la región de reclutamiento y serología del donador encontramos que para la Región 2 Sierra Nororiental se obtuvo una asociación entre ser seronegativo y haber sido reclutado en ella $\left(\chi^{2}=5.13\right.$, $p=0.008)$, mientras que para la Región 7 Tehuacán y Sierra Negra se encontró asociación entre ser seropositivo y haber sido reclutado en ella $\left(\chi^{2}=4.59, p=0.032\right)$.

Al comparar la seroprevalencia a T. cruzi con la región de origen del donador encontramos para la Región 2 Sierra Nororiental una asociación entre ser seronegativo y originario de la región $\left(\chi^{2}=6.83, p=0.008\right)$; lo mismo que, para la región 4 Angelópolis $\left(\chi^{2}=3.68\right.$ con $p=0.030)$; por el contrario, para la Región 7 Tehuacan y Sierra Negra se encontró una asociación entre ser seropositivo y originario de ésta $\left(\chi^{2}=5.87, p=0.015\right)$.

La relación de la seroprevalencia positiva a T. cruzi con la altitud de los municipios alcanzó significancia estadística marginal una $\left(\chi^{2}=2.39, p=0.120\right)$.

\section{Discusión}

La transfusión sanguínea es una de las vías de transmisión del T. cruzi y una de las acciones para controlarla es realizar un tamizaje en donadores de sangre para la detección de Ac anti-T. cruzi. ${ }^{2}$

El estado de Puebla fue considerado como de baja seroprevalencia a T. cruzi $(0.3 \%)$, de acuerdo con la Encuesta Nacional Seroepidemiológica en población abierta, en la cual se utilizó la técnica de HAI con dilución 1:8; tal seroprevalencia resultó ser de $0.0 \%$ cuando se consideraron dos pruebas serológicas ${ }^{16}$ y, de acuerdo con la Encuesta Centinela en Bancos de Sangre, se encontró de $1.8 \% .{ }^{10}$ En un estudio previo, en 1996, en 4263 donadores de sangre de la SSA se obtuvo una seroprevalencia de $0.8 \%$ empleando HAI e IFI, reactivos del Instituto de Diagnóstico y Referencia Epidemiológicos ${ }^{*}$ y en un estudio recientemente publicado se encontró una seroprevalencia que alcanzó $7.7 \%{ }^{14}$

En el actual trabajo obtuvimos una seroprevalencia a T. cruzi de $1.4 \%$. Seis de los puestos de sangrado tienen áreas de influencia netamente rurales (Hospitales Rurales Oportunidades), tres son del ámbito rural y suburbano (HGZ No. 5, 15 y 23) y uno recluta, en su gran mayoría, donadores de ámbito urbano.

Nuestros datos señalan que la distribución de la seroprevalencia por lugar de origen de los donadores es heterogénea, oscila desde $0.0 \%$ a $2.6 \%$ y está relacionada con el lugar de origen de los mismos, lo que indica posiblemente factores de riesgo diferentes según la zona de origen del donador.

* Gutiérrez-Rodríguez MS. Seroprevalencia de la enfermedad de Chagas en donadores de sangre en el estado de Puebla, de enero a octubre de 1996. Puebla, Puebla: Centro Estatal de la Transfusión Sanguínea, Secretaría de Salud del Estado de Puebla; 1996. Documento no publicado. 


\section{Cuadro III \\ Relación de La SEROPREVAlenCia A T. CRUZI, POR ORIGEN DEL DONADOR Y LA ALTITUd DE LOS MUNICIPIOS en donde habitan. Puebla, México, 2003}

\begin{tabular}{|c|c|c|c|c|c|}
\hline Nombre de la región & $\begin{array}{l}\text { Intervalo y promedio } \\
\text { de altitud (msnm) } \\
\text { de los municipios origen } \\
\text { de los donadores }\end{array}$ & $\begin{array}{l}\text { Seroprevalencia por } \\
\text { región de origen del donador, } \\
\text { por la técnica } \\
\text { de ELISA \% }\end{array}$ & $\begin{array}{l}\text { Relación de donadores } \\
\text { seropositivos y } \\
\text { seronegativos, } \\
\text { por región de origen }\end{array}$ & $\begin{array}{c}\text { M unicipios origen } \\
\text { de los donadores } \\
\text { seropositivos a T. cruzi } \\
\text { Nombre y altitud msnn }\end{array}$ & \\
\hline 1. Sierra N orte & $\begin{array}{c}190 \text { a } 2800 \\
1483\end{array}$ & $5 / 3771.3$ & 1:66 & $\begin{array}{l}1 \text { Pantepec } \\
1 \text { Fco Z Mena } \\
1 \text { Xicotepec de Juárez } \\
1 \text { Tlacuilotepec } \\
1 \text { Teotlalpan, C hignahuapan }\end{array}$ & $\begin{array}{r}625 \\
300 \\
1150 \\
1300 \\
2320\end{array}$ \\
\hline 2. Sierra N ororiental & $\begin{array}{c}700 \text { a } 2000 \\
1267\end{array}$ & $0 / 3830.0$ & & & \\
\hline 3.Valle de Serdán & $\begin{array}{c}1950 \text { a } 2600 \\
2340\end{array}$ & $3 / 1961.5$ & $1: 65$ & $\begin{array}{l}1 \text { A catzingo } \\
1 \text { Tecamachalco } \\
1 \text { Chichiquila }\end{array}$ & $\begin{array}{l}2150 \\
2060 \\
1700\end{array}$ \\
\hline 4. Angelópolis & $\begin{array}{c}1950 \text { a } 2600 \\
2375\end{array}$ & $0 / 2220.0$ & & & \\
\hline 5.Valle de Atlixco y Matamoros & $\begin{array}{c}1080 \text { a } 1850 \\
1075\end{array}$ & $5 / 3281.5$ & $1: 65$ & $\begin{array}{l}2 \text { Atlixco } \\
2 \text { Chietla } \\
1 \text { Izúcar de Matamoros }\end{array}$ & $\begin{array}{l}1850 \\
1000 \\
1300\end{array}$ \\
\hline 6. Mixteca & $\begin{array}{c}800 \text { a } 2000 \\
1352\end{array}$ & $3 / 1152.6$ & 1:38 & $\begin{array}{l}1 \text { Atexcal } \\
1 \text { Juan N Méndez } \\
1 \text { Chiautla de Tapia }\end{array}$ & $\begin{array}{l}1700 \\
2000 \\
1000\end{array}$ \\
\hline 7.Tehuacán y Sierra N egra & $\begin{array}{c}1100 \text { a } 2200 \\
1645\end{array}$ & $16 / 6452.4$ & $1: 40$ & $\begin{array}{l}11 \text { Tehuacán } \\
1 \text { Zozutla,Yehualtepec } \\
1 \text { Zinacatepec } \\
1 \text { Tepanco de López } \\
1 \text { Santiago Miahuatlán } \\
1 \text { Papalopa, Eloxochitlán }\end{array}$ & $\begin{array}{r}1640 \\
2050 \\
410 \\
1800 \\
1800 \\
1300\end{array}$ \\
\hline
\end{tabular}

La discordancia respecto al estudio recientemente publicado que señala hasta $7.7 \%$ de seroprevalencia general para el estado, ${ }^{14}$ pudiera deberse a la estandarización de los procedimientos utilizados y a la posibilidad de considerar como positivos aquellos sueros reactivos en los límites del valor de corte o la llamada zona gris. El efecto de la cepa de T. cruzi para la producción de antígeno parece no ser una variable crítica, ya que en estudios recientes en los que se utilizaron como fuente de antígeno 15 cepas mexicanas procedentes de distintas zonas geográficas del país no observamos discordancia entre ellas.*

\footnotetext{
* Monteón-Padilla VM. Uso de distintos aislados mexicanos de Trypanosoma cruzi en diagnóstico de la enfermedad de Chagas. Mexico, DF: Instituto Nacional de Cardiología/Centro de Investigación de Enfermedades Tropicales, Campeche; 2005. Documento no publicado.
}

La seroprevalencia obtenida en este trabajo fue de $1.4 \%$ (37/2 489), dato que es semejante al del estudio centinela en bancos de sangre, que informó de 1.8\% general para el estado; ${ }^{10}$ esto posiblemente se deba a que los procedimientos utilizados entre los dos laboratorios han sido previamente validados, observando valores de índice Kappa por arriba del 0.8, a pesar de utilizar técnicas diferentes con antígenos de cepas diferentes. ${ }^{17}$ En estudios previos se han ido determinando las posibles reacciones cruzadas con otras enfermedades, principalmente con los anticuerpos a Toxoplasma gondii, los cuales no han presentado reactividad; con Leishmania sp y Trypanosoma rangeli hasta de $10 \%$ y en valores cercanos al valor de corte. En este estudio el valor de corte fue de 0.240 y el promedio general del nivel de anticuerpos de seropositivos fue de DO 0.581.

Al comparar los niveles de anticuerpos anti-T. cruzi y la procedencia de los donadores no encontramos diferencia estadísticamente significativa, a pesar de ver- 
se una tendencia de mayor nivel de anticuerpos en los procedentes de otros estados del país.

Al comparar los resultados de seroprevalencia con las otras pruebas que son obligatorias para los bancos de sangre observamos que los donadores estudiados presentaron la reactividad más alta hacia VHC $(1.5 \%)$ y VHBc $(1.1 \%)$, mientras para VIH y para AgVHBs estuvieron por abajo de $0.6 \%$. Estos datos sugieren fuertemente que el escrutinio para anti-T. cruzi debe ser incluido en el tamizaje de los donadores de banco de sangre, incluso en zonas donde la seroprevalencia para esta infección sea considerada baja o ausente, ya que el movimiento de la población es un elemento dispersante de este riesgo, al detectar regiones que reclutan a un alto porcentaje de donadores de sangre que son originarios de las otras regiones y de otros estados, como las Regiones 6 Mixteca, 4 Angelópolis y la 7 Tehuacán Sierra Negra (cuadro I). Al analizar el aporte de seropositivos provenientes de otros estados tenemos que $5 / 223(2.2 \%)$ fueron positivos y que procedían de los estados de Morelos, Oaxaca y Veracruz, donde se ha reconocido la existencia de transmisión de T. cruzi al humano.

En la Región 7 Tehuacán Sierra Negra se observó que 645 donadores reclutados fueron originarios de la misma (94\%), con 16 seropositivos, y dos de ellos procedían del estado de Oaxaca; esto nos indica que posiblemente se infectan en su región de origen y un bajo porcentaje proviene de fuera, como el estado de Oaxaca, el cual tiene zonas endémicas reconocidas. ${ }^{16} \mathrm{La}$ asociación entre seropositividad y ser originario de esta zona sugiere que las unidades de sangre provenientes de la Región 7 son las de más alto riesgo para la probable transmisión de T. cruzi por transfusión.

La edad promedio de nuestros donadores de sangre fue de 31.4 años. Al buscar asociación entre edad y seropositividad encontramos que para los sujetos entre la cuarta y quinta década de vida ( 2.89 y $8.53 \%$ de seropositividad, respectivamente) sí existió asociación con una $\chi^{2}=12.88$ con $p=0.003$ y $\chi^{2}=9.33$ con $p=0.021$ Este mismo patrón se ha observado en estudios de población abierta, donde la seroprevalencia aumenta considerablemente en la cuarta y quinta década de vida. ${ }^{13,16}$

El habitar en municipios ubicados a menos de 2200 msnm y seropositividad a T. cruzi alcanzó significancia estadística marginal; es posible que este resultado se deba a que $85.8 \%$ de los donadores habitaron en municipios ubicados a una altitud menor de 2200 msnm. Además, la Región 1 Sierra Norte, tiene la superficie territorial más irregular (190 a los $2800 \mathrm{msnm}$ ), donde encontramos donadores seropositivos a T. cruzi desde los 190 msnm, en los municipios de Francisco Z Mena, hasta los 2 350, en Chiganahuapan.

A todas estas variables hay que agregar también la existencia de triatominos; así, tenemos que hay antecedentes de su presencia en la mayoría de las regiones estudiadas, incluso se les ha identificado parasitados. ${ }^{12,13,18,19}$ El hallazgo de una mayor seroprevalencia en las Regiones 6 y 7 podría deberse a su clima, éste es semiseco, de templado a cálido con lluvias en el verano y escasas a lo largo del año en las dos regiones, y quizás sea favorable a los triatominos vectores esparcidos en la zona -Triatoma barberi, Meccus bassolsae y Meccus pallidipennis-. ${ }^{*}$ Mientras que el vector esparcido en la Región 2 es Triatoma dimidiata. ${ }^{18}$ La presencia del vector, que es el principal mecanismo de transmisión de la infección, parece por sí solo no ser suficiente para esperar también una infección alta en los humanos. Estos datos sugieren que debe conocerse más sobre los hábitos de los triatominos vectores y su relación con el humano.

Estos datos señalan que en el estado de Puebla existe el riesgo para la transmisión de la infección de T. cruzi por transfusión sanguínea y, de no realizarse adecuadamente el tamizaje, de diseminar la infección a través de este mecanismo.

En conclusión, la seropositividad a T.cruzi en donadores de banco de sangre, en el estado de Puebla, pudiera ser considerada entre 1.1 a $1.8 \%$, la cual es comparable con la observada para VHC con 1.5\%. Las regiones 6 Mixteca y la 7 Tehuacán y Sierra Negra son las de más alto riesgo, con seroprevalencia de hasta $2.6 \%$. Además, se identificaron donadores seropositivos en localidades desde los $190 \mathrm{msnm}$ hasta los 2320 msnm.

\section{Agradecimientos:}

A la M.C. Martha Ballinas Verdugo del laboratorio de Inmunoparasitología del Instituto Nacional de Cardiología. A la Q.F.B. Herminia Juárez Zamora y a la Lab. Julia Inés Ruiz Conde del banco de sangre del Hospital de Especialidades del Centro Médico Nacional Manuel Avila Camacho, del Instituto Mexicano del Seguro Social, por su participación en la identificación de las muestras séricas procedentes de los nueve puestos de sangrado del IMSS, Puebla. Asimismo a la doctora

\footnotetext{
* Zumaquero-Ríos JL. Distribución vectorial de triatominos (Hemiptera-Reduviridae en el estado de Puebla. Puebla, Puebla: Escuela de Biología, Benemérita Universidad Autónoma de Puebla; 2005. Documento no publicado.
} 
María del Socorro Gutiérrez Rodríguez, directora del Centro Estatal de la Transfusión Sanguínea, de la Secretaría de Salud, Puebla, por su valiosa información.

\section{Referencias}

1. Bijovsky AT, Milder RV.Abrahamsohn IA, Sinhorini IL, Mariano M.The influence of limphatic drainame in experimental Trypanosoma cruzi infection. Acta Trop 1984; 41:207-214.

2. Pinto-D ias JC,W endel S, Brener Z, C amargo ME, Rassi A. Epidemiology of Chagas disease. Sao Paulo: Ed ISBT; 1992:1-5. 3. Goldsmith RS, Zárate R, Kagan I, Cedeño-Ferrerira J, GalindoVasconcelos M, Paz EA. El potencial de la transmisión en la enfermedad de Chagas por transfusión sanguínea: hallazgos serológicos entre donadores en el estado de 0 axaca. Salud Publica Mex 1978; 20(4):439444.

4. Salazar-Schettino PM, Barrera M, Bucio MI.Transmisión de Trypanosoma cruzi por transfusión sanguínea. Primer caso humano en México. Rev Mex Patol Clin 1989; 36:57-59.

5. Secretaría de Salud N O M-003-SSA 2-1993. Para la disposición de sangre humana y sus componentes con fines terapéuticos. México, DF: Diario 0 ficial de la Federación, 8 de diciembre de 1993.

6. Trujillo-Contreras F, Lozano-Kasten F, Soto-Gutiérrez MM, Hernández-Gutiérrez R. Prevalencia de infección a Trypanosoma cruzi en donadores de sangre en el estado de Jalisco, México. Rev Soc Bras Med Trop 1993;26:89-92.

7. Ramos-Echeverría A, Monteón-PadillaVM, Reyes-López PA. D etección de anticuerpos contra Trypanosoma cruzi en donadores de sangre. Salud Publica Mex 1993;35(1):56-64.

8. Monteón-PadillaVM, Hernández-Becerril N , G uzmán-Bracho C, Rosales-Encinas Jl, Reyes-López PA. A merican tripanosomiasis (Chagas' $D$ isease) and blood banking in Mexico City. Seroprevalence and its potential transfusional transmission risk. Arch Med Res 1999;30:393398.

9. Rangel $H, G$ atica R, Ramos C. D etection of antibodies against Trypanosoma cruzi in donors from a blood bank in Cuernavaca, Morelos, Mexico.Arch Med Res 1998;29:79-82.
10. Guzmán-Bracho C, G arcía-García L, Floriani-Verdugo J, GuerreroMartínez S,Torres-Cosme M, Ramírez-Melgarejo et al. Riesgos de transmisión de T. cruzi por transfusión de sangre. Rev Panam Salud Publica 1998; 4(2):590-601.

11. Cerisola JA, Rabinovich A,Alvarez M, Di Corleto CA, Pruneda J. Enfermedad de Chagas y la transfusión de sangre. Bol 0 ficina Sanit Panam 1972; 63:203-221.

12. Pérez-Fuentes $R$, Sánchez-Guillén $M C$, $G$ onzález-Alvarez $C$, Monteón-PadillaVM, Reyes-López PA, Rosales-Encinas Jl. Humoral nitric oxide levels and antibody immune response of symptomatic an indeterminate $C$ hagas disease patientes to commercial and autochthonous Trypanosoma cruzi antigen. Am J Trop Med Hyg 1998; 58(6):715-720.

13. Sosa-Jurado F, Zumaquero-Ríos JL, Reyes PA, C ruz-GarcíaA, Guzmán-Bracho C, Monteón VM. Factores bióticos y abióticos que determinan la seroprevalencia de anticuerpos contra Trypanosoma cruzi en el municipio de Palmar de Bravo, Puebla, México. Salud Publica Mex 2004; 46: 39-48.

14. Sánchez-G uillén MC, Barnabé C, Juegan JF,Tibayrenc M, Velásquez Rojas M, Martínez-Munguía J. High prevalence anti-Trypanosoma cruzi antibodies, among blood donors in the State of Puebla, a non-endemic area of Mexico. Mem Inst 0 swaldo Cruz 2002; 97 (7):947-952.

15. Secretaría de Comunicaciones y Transportes del Estado de Puebla. Siete regiones económicas del estado de Puebla, México. Plan Estatal de Desarrollo Regional 1999-2005. Puebla, México: SC TEP; 2003.

16.Velasco-C astrejón O,Valdespino JL,Tapia-C onyer R, Salvatierra B, Guzmán-Bracho C, Magos C et al. Seroepidemiología de la enfermedad de Chagas en México. Salud Publica Mex 1992;34(2):186-196.

17. Monteón VM, Guzmán-Bracho C, Floriani-Verdugo J, RamosEchevarría A, Velasco-Castrejón 0, Reyes PA. Diagnóstico serológico de la enfermedad de Chagas: autosuficiencia y concordancia interlaboratorios. Salud Publica Mex 1995; 37(3):232-235. 18. Sandoval RCA, Zumaquero RJL, Linares G. Infección natural con Trypanosoma cruzi en triatominos (Hemiptera: Reduviidae:Triatominae), vectores de la enfermedad de Chagas en San Antonio Rayón, Jonotla, Puebla, México. Rev Tecnociencia Panama 2004; 6(1):60-66.

19.Tay-Zavala J, Sánchez-Vega JT, Robert-Guerrero L,Alonso-Guerrero T, Romero-C aballero R. N uevas localidades con triatominos infectados por Trypanosoma cruzi en la República Mexicana. Bol Chil Parasitol 1996; 51:49-53. 